\title{
PTEN overexpression suppresses proliferation and differentiation and enhances apoptosis of the mouse mammary epithelium
}

\author{
Joëlle Dupont, ${ }^{1}$ Jean Pierre Renou, ${ }^{2}$ Moshe Shani, ${ }^{2}$ Lothar Hennighausen, ${ }^{2}$ \\ and Derek LeRoith ${ }^{1}$ \\ ${ }^{1}$ Section on Molecular and Cellular Physiology, Clinical Endocrinology Branch, and \\ ${ }^{2}$ Laboratory of Genetics and Physiology, National Institute of Diabetes and Digestive and Kidney Diseases, NIH, \\ Bethesda, Maryland, USA
}

\begin{abstract}
The phosphatase PTEN regulates growth, adhesion, and apoptosis, among many other cell processes. To investigate its role during mouse mammary gland development, we generated MK-PTEN, a transgenic mouse model in which human PTEN is overexpressed in ductal and alveolar mammary epithelium during puberty, pregnancy, lactation, and involution. No obvious phenotype was observed in mammary tissue of pubescent virgin mice. However, MK-PTEN females could not lactate normally, and $\sim 30 \%$ of pups died, with survivors exhibiting growth retardation. Transgenic offspring nursed by wild-type foster mothers, conversely, developed normally. This phenotype is consistent with a reduced number of alveolar epithelial cells due to a decrease in cell proliferation and an increase in apoptosis. Using mammary-enriched cDNA microarrays, we identified several genes that were preferentially expressed in MK-PTEN mammary tissue, including the IGF-binding protein-5 (Igfbp5) gene, and others whose expression was reduced, including the genes for c-Jun amino-terminal kinase. Secretory epithelial cell differentiation was impaired, as measured by the expression of specific milk protein genes. MK-PTEN mice also exhibited a 50\% decrease in the phosphorylation state of Akt. Taken together, these results suggest that PTEN controls mammary gland development and, consequently, lactation.
\end{abstract}

J. Clin. Invest. 110:815-825 (2002). doi:10.1172/JCI200213829.

\section{Introduction}

The mammary gland is composed of an epithelium that synthesizes and secretes lipid and milk proteins. It develops primarily postnatally, according to distinct stages that are largely controlled by steroids, peptide hormones, and cell matrix interactions (1). During puberty, ductal elongation and branching are induced by hormones, such as estrogen, progesterone, and growth hormone (2). During pregnancy, alveoli are formed and produce milk during lactation under the control of insulin, hydrocortisone, and prolactin (3). Upon weaning, the majority of the secretory epithelium undergoes apoptosis in a process called involution. Development of the

Received for publication July 25, 2001, and accepted in revised form July 9, 2002.

Address correspondence to: Derek LeRoith, Clinical Endocrinology Branch, NIDDK, Room 8D12, Building 10, NIH, Bethesda, Maryland 20892-1758, USA. Phone: (301) 496-8090; Fax: (301) 480-4386; E-mail: Derek@helix.nih.gov.

Joëlle Dupont's present address is: Physiologie de la

Reproduction et des Comportements, Université Francois Rabelais de Tours, Nouzilly, France.

Conflict of interest: No conflict of interest has been declared. Nonstandard abbreviations used: mouse mammary tumor virus (MMTV); long-terminal repeat (LTR); whey acidic protein (WAP); phosphatidylinositol 3-kinase (PI3K); mitogen-activated protein kinase (MAPK); c-Jun $\mathrm{NH}_{2}$-terminal kinase (JNK); stress-activated protein kinase-associated protein-1 (SAPK-1); IGF-binding protein-5 (IGFBP-5); wild-type (WT); high-mobility group protein I isoform C (HMG-IC); glucocorticoid-induced leucine zipper (GILZ); cyclin-dependent kinase (CDK). mammary gland also requires other peptide growth factors, such as the IGFs, EGF, PDGF, and their associated receptors. Some evidence supports a role for the stromal EGF receptor and its ligands in mammary morphogenesis $(4,5)$. Several studies suggest that the IGFs are critical for normal postnatal growth and differentiation of mammary epithelium (6). Experiments in transgenic mice show that overexpression of IGF-1 driven by the mouse mammary tumor virus-long-terminal repeat (MMTVLTR) results in precocious alveolar development when expressed in virgin mammary glands (7). Overexpression of IGF-1 can also delay involution when expressed from the whey acidic protein (WAP) gene promoter $(8,9)$.

PTEN (phosphatase and tensin homolog; ref. 10) is a lipid and protein phosphatase. Mutations in PTEN have been found in a wide variety of human tumors, including breast, brain, prostate, and endometrium (11). Evidence suggests that the tumor suppressor status of PTEN relies on its normal cellular activity as a lipid phosphatase. This activity reduces the cellular levels of PIP3 and thus antagonizes the action of the phosphatidylinositol 3-kinase (PI3K) and inhibits a number of signaling pathways, including protein kinase B (PKB/Akt), as well as various cellular processes, including proliferation, cell survival, and apoptosis (reviewed in ref. 12). However, it remains unclear whether PIP3 is the only major substrate for PTEN in vivo, because dephosphorylation of protein substrates including the adapter protein 
Shc and FAK and inhibition of the mitogen-activated protein kinase (MAPK) pathway have also been reported $(13,14)$.

Stimulation of the IGF-1, insulin, EGF, and PDGF receptor tyrosine kinases activates both PI3K/Akt and MAPK pathways. These two signaling pathways can also be activated by estrogen (15), prolactin (16), and growth hormone (17), which all regulate mammary gland development. Several studies have shown that in the mammary gland epithelium, MAPK and PI3K/Akt regulate cell proliferation (18), cell survival (19), and apoptosis (20). When a constitutively active mutant of Akt was expressed specifically in the mouse mammary gland, delays in involution and in the onset of apoptosis were observed $(21,22)$. Thus, further characterization of proteins that regulate the PI3K/Akt and the MAPK signaling pathways is important for understanding both the regulation of normal mammary gland development and the process of mammary tumorigenesis.

In the present study, we have examined the role of PTEN in mammary gland development using transgenic mice in which PTEN is overexpressed specifically in mammary epithelium using the MMTV-LTR. We show that the overexpression of PTEN decreases mammary epithelial cell proliferation and increases apoptosis, thereby resulting in the formation of alveolar hypoplasia. In addition we demonstrate that functional differentiation of mammary epithelium is impaired. Consequently, the process of lactation is affected, and the newborn pups either die or exhibit growth retardation. At the molecular level, we show that the overexpression of PTEN in mammary gland significantly inhibits the Akt signaling pathway, but that it does not affect the MAPK pathway. Furthermore, using oncogene- or mammary-enriched cDNA microarrays, we identify new target genes for PTEN, including the c-Jun $\mathrm{NH}_{2}$-terminal kinase/stress-activated protein kinase-associated protein-1 (JNK/SAPK-1) and the IGF-binding protein-5 (igfbp-5) genes, that could explain the reduction of the cell proliferation and the increase in the apoptosis of the MK-PTEN epithelial cells, respectively.

\section{Methods}

Generation of MK-PTEN transgenic mice. The MMTV-LTR promoter was used to generate transgenic mice expressing PTEN specifically in the mammary gland. The MMTV-KCR vector was kindly provided by Sophia Y. Tsai (Baylor College of Medicine, Houston, Texas, USA) (23). A 1,218-bp blunt-ended EcoRI fragment of the human PTEN cDNA, kindly provided by Kaz Matsumoto (National Institute of Dental and Craniofacial Research, $\mathrm{NIH}$ ), was cloned into the blunt-ended EcoRI site of the MMTV-KCR vector. The MMTV-KCR-PTEN plasmid was then digested with Acc65I and XbaI to liberate a 4,029-bp fragment. This fragment was microinjected into fertilized eggs derived from C57BL/6J mice. These eggs were implanted into CD-1 foster mothers, and integration of the transgene in the offspring was assessed by Southern blot and PCR analyses, as described below.
Genotyping and animals. Genotypic analyses by both PCR and Southern blot were performed on genomic DNA isolated from the tails of potential founder mice. Primers used for the detection of the transgenic product were GLE3-F (5'-TGGTTGTTGTGCTGTCTCATC-3') and PTEN-R (5'-CAAAAGGATATTGTGCAACTC- $\left.3^{\prime}\right)$. Southern blots were performed using a 436-bp fragment of the PTEN cDNA as a probe for transgene detection on genomic DNA that had been digested with BamHI. Similar phenotypes of mammary gland hypoplasia were observed in two independent transgenic lines. Thus, the results reported are primarily from the transgenic line 7011, unless otherwise specified. Mice were kept under a 12-hour light/dark cycle and were fed standard diets. Procedures were conducted in compliance with the guidelines of the Animal Care and Use Committee of the National Institute of Diabetes and Digestive and Kidney Diseases (NIH). To determine the pregnancy stage, the mice were mated and inspected for the presence of vaginal plugs in the mornings. The day of the vaginal plug was counted as day 0 of pregnancy.

Isolation and primary culture of mammary epithelial cells. Primary mammary epithelial cells were prepared from wild-type (WT) and MK-PTEN mice pregnant for 16-19 days, as described previously (24). Cells were resuspended in DMEM with phenol red containing $10 \%$ FBS, 100 units $/ \mathrm{ml}$ penicillin, $100 \mathrm{mg} / \mathrm{ml}$ streptozotocin, $10 \mathrm{ng} / \mathrm{ml}$ EGF (Upstate Biotechnology Inc., Lake Placid, New York, USA), and $5 \mu \mathrm{g} / \mathrm{ml}$ insulin (Sigma-Aldrich, St. Louis, Missouri, USA). After 48 hours, the medium was removed and fresh medium was added with or without the IGFBP-5-blocking peptide for 24 hours. This IGFBP-5-blocking peptide (25) was kindly provided by D.R. Clemmons (Department of Medicine, University of North Carolina, Chapel Hill, North Carolina, USA).

Determination of caspase-3 activity. We determined caspase- 3 activity by measuring the absorbance at $405 \mathrm{~nm}$, following cleavage of the synthetic substrate, Ac-AspGlu-Val-Asp-chromophore $p$-nitroaniline (Ac-DEVDpNA). Briefly, mammary epithelial cells from WT and MK-PTEN mice incubated in the presence or absence of the IGFBP-5-blocking peptide were lysed on ice for 10 minutes in cell lysis buffer provided in the Clontech ApoAlert Caspase-3 colorimetric assay kit (Clontech Labs, Palo Alto, California, USA). Lysates were then incubated with $50 \mu \mathrm{M}$ Ac-DEVD pNA in the reaction buffer containing 0.1 M HEPES, 20\% glycerol, $10 \mathrm{mM}$ DTT, and protease inhibitors. The lysates were maintained at $37^{\circ} \mathrm{C}$ for 1 hour and then analyzed in an ELISA plate reader. Levels of relative $p$-nitroaniline were normalized against the protein concentration of each extract.

RNA isolation and Northern blots. Total RNA from the left inguinal mammary gland (gland number 4) was extracted at the indicated times of development using TRIzol reagent according to the manufacturer's instructions (Invitrogen Corp., Carlsbad, California, USA). Twenty micrograms of total RNA was separated in 1.5\% 
formaldehyde gels and blotted on nylon membranes. Expression of milk protein RNA and keratin 18 was analyzed as described previously (26). Expression of PTEN was analyzed using the entire 1.2-kb human PTEN cDNA as a probe. RNA levels were quantified using a PhosphoImager apparatus (Fujifilm Medical Systems USA Inc., Stamford, Connecticut, USA). The integrity of different transcripts was assessed using the human RNA $18 \mathrm{~S}$ probe from Ambion Inc. (Austin, Texas, USA).

RT-PCR analysis of mRNAs. Total RNA from mammary glands of WT and MK-PTEN mice (day 2 of lactation) was extracted as described above. One microgram of DNase-treated RNA of each group (WT and MK-PTEN) was reverse-transcribed using $2.5 \mathrm{U} / \mathrm{ml}$ of murine leukemia virus reverse transcriptase, $2.5 \mathrm{mM} \mathrm{MgCl} 2,1$ $\mathrm{mM}$ dNTP, $2.5 \mathrm{mM}$ oligo-dT, and $1 \mathrm{U} / \mathrm{ml}$ RNase inhibitor. The same reverse transcription products were subjected to PCR in standard conditions in the presence of specific primers: sense PTEN, $5^{\prime}$-GAAGATATATTCCTCCAATTC- $3^{\prime}$; antisense PTEN, 5' -CTTGATCACATAGACTTCCAT-3'; sense WAP, $5^{\prime}$-GACACCGGTACCATGCGTTG-3'; antisense WAP, $5^{\prime}$-TAGCAGCAGATTGAAAGCATT- ${ }^{\prime}$; sense IGFBP-5, 5' ${ }^{\prime}$-TGTGGCTGCTGCATGACTTG- $3^{\prime}$; antisense IGFBP-5, 5' ${ }^{\prime}$-GAACTCCTGGAGGGAAGCTT- ${ }^{\prime}$; sense $\varepsilon$-casein, $5^{\prime}$-TCTGACTTGCCTTTTGGCCG- $3^{\prime}$; antisense $\varepsilon$-casein, $5^{\prime}$-AAACTGTGGCCAGGTGATCT- $3^{\prime}$; sense carbonic anhydrase 3 , 5' ${ }^{\prime}$-ACAATGGTCCTGAGCACTGG-3'; antisense carbonic anhydrase 3 , 5'-GTGAAGC-TCAGC-AGCATACT-3'; sense cdc2, 5'-TCAGCAGCATCTGCATCACT-3'; antisense cdc2, 5'-AGATCGGCCAAGGCACATTC- ${ }^{\prime}$; sense $\beta$-actin, $5^{\prime}$-ACGGAACCACAGTTTATCATC- $3^{\prime}$; antisense $\beta$-actin, 5'-CCATATCAACTTCTGACCCTG-3'; sense JNK/SAPK-1, $5^{\prime}$-TACCAACCATGTGGCTTGGA-3'; antisense JNK/ SAPK-1, $5^{\prime}$-TTGCAAGACACCATAAGAGC- $3^{\prime}$. The identity of all PCR products was verified by sequencing.

Western blot analysis. Proteins from mammary glands were extracted and processed for immunoblotting exactly as described previously (27). The resulting immunoreactive bands were subjected to densitometry and quantitated with MacBas V2.52 software (Fujifilm Medical Systems USA Inc.).

Antibodies. Monoclonal anti-Actin (Clone AC) was obtained from Sigma-Aldrich. Rabbit polyclonal antibodies to phospho-Akt (Ser473), Akt, phospho-Erk1/2 (Thr202/Tyr204), and PTEN were purchased from New England Biolabs Inc. (Beverly, Massachusetts, USA). The rabbit polyclonal antibodies to Erk1 (C16) and IGFBP-5 (C18) were purchased from Santa Cruz Biotechnology Inc. (Santa Cruz, California, USA). All antibodies were used at a dilution of 1:1,000 in Western blotting.

Phosphatase assay. Mammary gland protein extracts containing $500 \mu \mathrm{g}$ of protein were immunoprecipitated overnight using a rabbit polyclonal anti-PTEN antibody (C20) developed against the ${ }^{384}$ DSDPENEPFDEDQHTQITKV $^{403}$ region of the human PTEN molecule (Zymed Laboratories Inc., South San Francisco, California, USA), and the phosphatase assay was performed exactly as described previously (28). The absorbance at $630 \mathrm{~nm}$ was recorded in an ELISA plate reader. A standard curve was performed with each assay, and the amount of free phosphate was calculated from the standard curve data.

Whole mounts, histology, and immunobistochemistry. Whole-mount analyses were performed as previously described (26). To prepare mammary gland sections, the left inguinal glands were fixed for 4 hours in $4 \%$ phosphate-buffered paraformaldehyde, then transferred to $70 \%$ ethanol. Tissues were embedded in paraffin and then cut into $5-\mu \mathrm{m}$ sections. PTEN immunostaining was performed as previously described (29) using the $\mathrm{mAb} 6 \mathrm{H} 2.1$. This antibody was raised against the last $100 \mathrm{C}$-terminal amino acids of human PTEN. Specificity and characterization of the PTEN antibody $6 \mathrm{H} 2.1$ have been previously demonstrated by Western blot and immunohistochemistry on cell lines with known PTEN expression status (29). IGFBP-5 immunostaining was performed in the same conditions as the PTEN immunostaining. Incubating the sections with normal serum in the absence of antibody resulted in no signal (data not shown).

Cell proliferation and cell death assays. Two hours before sacrifice, mice were injected with $20 \mu \mathrm{l} / \mathrm{g}$ of body weight of cell labeling reagent (Amersham Biosciences, Piscataway, New Jersey, USA). Mammary glands were prepared and fixed in $4 \%$ paraformaldehyde for 4 hours. The tissues were then embedded in paraffin. For detection of incorporated $\mathrm{BrdU}, 5-\mu \mathrm{m}$ sections were processed according to the manufacturer's instructions (Amersham Life Science). On adjacent sections from the same samples, apoptotic cells were detected by TUNEL assay with ApopTag Plus Peroxidase, according to the manufacturer's instructions (Intergen Co., Purchase, New York, USA). Between 2,000 and 3,000 epithelial cells were counted from three to four different sections of a sample, and the averages of percentages of labeled cells were calculated.

Microarray analyses. A cDNA array (mammochip) containing 3,500 genes assembled from various mammary gland cDNA libraries (NbMMG, NMLMG, CGAP Mam1 to -6$)$ was generated and printed on glass slides (K. Miyoshi and L. Hennighausen, unpublished observations). In addition, the National Cancer Institute oncochip containing 2,600 genes was used. RNA was prepared from mammary tissue from MK-PTEN and control mice at day 2 after parturition (about 12 weeks of age). For each experiment, samples from two different mice were pooled. For fluorescence labeling, $20 \mu \mathrm{g}$ of total RNA was reverse-transcribed in the presence of 300 U of SstII (Life Technologies Inc., Rockville, Maryland, USA) and labeled with cy3-dUTP and cy5-dUTP (NEN Life Science Products Inc., Boston, Massachusetts, USA). Samples were combined, purified, and concentrated with Microcon YM30 columns (Millipore Corp., Bedford, Massachusetts, USA). Slides were prehybridized for 1 hour and hybridized overnight at $42^{\circ} \mathrm{C}$ in $25 \%$ formamide (30). Slides were washed as described (31) and scanned on a GenePix scanner (Axon Instruments Inc., Foster City, California, USA). The images were analyzed by GenePix Pro 3.0 (Axon Instruments Inc.). Each 


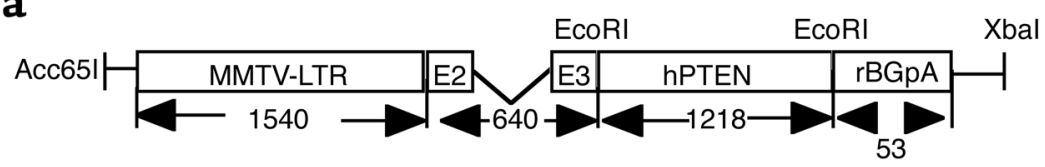

b

Southern blot

PCR

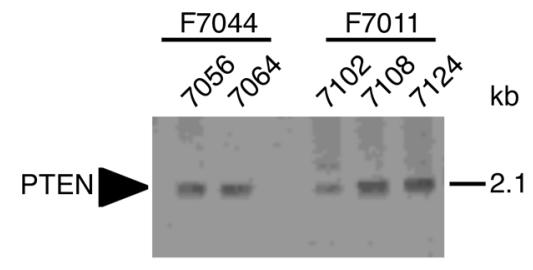

$\mathrm{kb}$
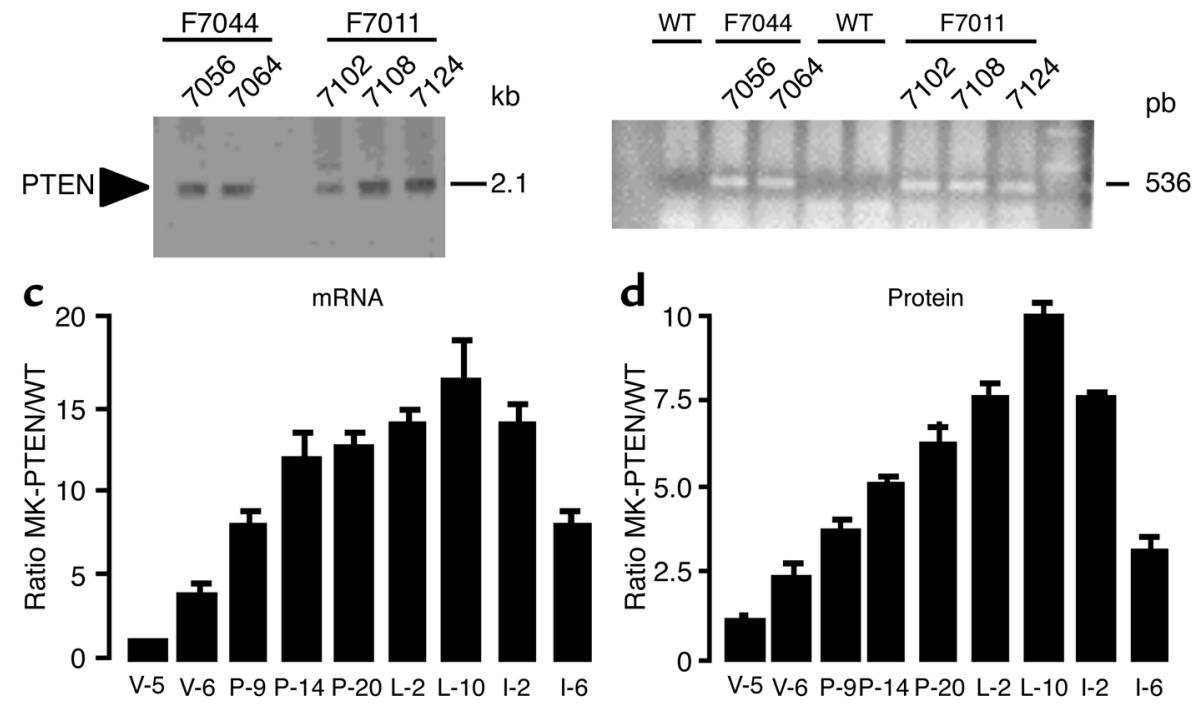

\section{Figure 1}

(a) Schematic of the construct used to generate MK-PTEN mice. (b) Southern blot and PCR show transmission of the transgene to the germline. Left: the presence of the $2.1-\mathrm{kb}$ transgene is indicated by the arrow. Right: the 536-bp product corresponds to PTEN. (c) mRNA and (d) protein profile expression of PTEN throughout the development of the mammary gland in MK-PTEN and WT mice. (c) Northern blot analysis was performed using total RNA isolated from mammary gland of WT and MK-PTEN mice at various stages of development. The blot was first hybridized with the full-length hPTEN probe followed by a control $18 \mathrm{~S}$ probe (data not shown). The ratio of MK-PTEN expression levels to that in WT mice, as normalized to 18S RNA levels, is shown on the graph. Results represent mean \pm SD for four MK-PTEN and four WT mice at each stage. (d) Protein extracts from mammary glands of MK-PTEN and WT mice at various stages were subjected to immunoblotting for PTEN. Samples contained equal levels of protein, as confirmed by reprobing each membrane with an anti- $\alpha$-actin antibody (data not shown). The ratio of PTEN protein level between MK-PTEN and WT mice at various stages of mammary development is represented as the mean \pm SD for four MK-PTEN and four WT mice at each stage. V-5 and V-6, virgin 5 and 6 weeks; P-9, P-14, and P-20, pregnancy 9, 14, and 20 days; $\mathrm{L}-2$ and $\mathrm{L}-10$, lactation 2 and 10 days; $\mathrm{I}-2$ and $\mathrm{I}-6$, involution 2 and 6 days.

experiment was repeated with the cy3-dUTP and cy5dUTP labeling switched. The data were analyzed with the mAdb tools of the National Cancer Institute-Center for Information Technology $\mu$ Array Center (32) with some modifications. The differentially expressed genes were selected by signal intensity fivefold higher than background in at least one channel and an expression ratio of 3:0.3 or higher. These criteria were also used in the experiments with the labels reversed.

Statistical analyses. Results are expressed as the mean \pm SEM. Statistical analysis of the data was performed using a Student's $t$ test.

\section{Results}

Generation of transgenic mice overexpressing PTEN specifically in the mammary gland. To determine the role of PTEN in the developing mammary gland, we overexpressed the human PTEN cDNA transgene (hPTEN) specifically in the mammary epithelium using the MMTV-LTR. The transgenic construct used is shown in Figure 1a. Six founders with transgene integration were obtained. A representative Southern blot and PCR derived from BamHI-digested genomic DNA are shown for two founders in Figure 1b. Of these six founders, five transmitted the transgene through the germline. These founders were then bred with WT mice to generate females to test the expression of PTEN.

Expression of PTEN throughout the development of the mammary gland in MK-PTEN and WT mice. We next analyzed each of the transgenic lines for the expression of PTEN. Total RNA was isolated from biopsies of inguinal mammary glands (number 4 ) from transgenic and WT littermates 10 days after the onset of lactation, when the MMTV-LTR has been shown to be highly active. In the mammary glands, we detected PTEN as a major transcript of $2.5 \mathrm{~kb}$ and two other mRNA species of 5 and $3 \mathrm{~kb}$, the latter two being weakly expressed (data not shown). Two founders, F7011 and F7044, highly expressed PTEN at day 10 of lactation, as compared with the WT mice. The profile of PTEN mRNA expression during the development of the mammary gland was determined in the founder F7011 (Figure 1c). In MK-PTEN mice, the level of PTEN mRNA progressively increased, as compared with WT littermates, from the age of 6 weeks (virgins), through the beginning of puberty and throughout pregnancy, peaking at 


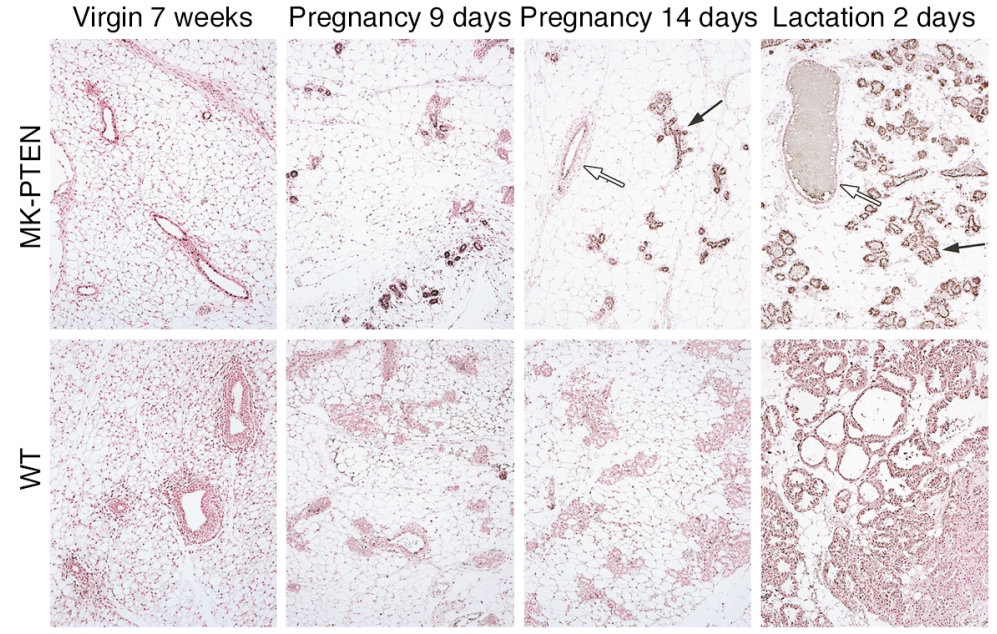

Figure 2

Immunohistochemical localization of PTEN in the mammary gland of MK-PTEN and WT mice. Sections from mammary glands removed from virgin, 9-day-pregnant, 14-daypregnant, and 2-day-lactating mice were analyzed by immunohistochemistry as described in Methods. PTEN-expressing lobuloalveoli and ductal epithelial cells are indicated by black arrows and white arrows, respectively.

day 10 of lactation and then decreasing during involution of the mammary gland (Figure 1c). These findings are in agreement with the hormonally regulated expression profile of the MMTV-LTR (23).

To determine whether the differences in mRNA were also reflected at the level of protein, we performed Western blot analyses on mammary gland extracts prepared from MK-PTEN and WT female mice at different stages of development. In 3- and 5-week-old virgins, two bands of approximately 54 and $58 \mathrm{kDa}$ in size were detected in both WT and MK-PTEN mice. These two bands are also detected when using two other PTEN antibodies, suggesting that they are specific and correspond to two isoforms of the PTEN protein (data not shown). At the age of 6 weeks, the level of PTEN protein is about twofold higher in MK-PTEN than in WT mice (Figure 1d). This difference between transgenic and WT mice progressively

\section{Figure 3}

PTEN phosphatase activity and phosphorylation state of Akt and MAPK in mammary glands of MK-PTEN and WT mice. (a) Mammary gland protein extracts from MK-PTEN and WT mice (500 $\mu \mathrm{g}$ protein per sample) were subjected to immunoprecipitation with the PTEN polyclonal C20 antibody, and the phosphatase activity was measured as described in the Methods. The phosphatase activity in MK-PTEN and WT is represented as mean \pm SD in the graph $(n=4$ MK-PTEN and WT mice for each group except in virgin and involution, where $n=2$ mice in each group). ${ }^{*} P<0.05,{ }^{*}{ }^{*} P<0.001$. (b) Akt and Erk1/2 activation in mammary gland extracts from MK-PTEN and WT mice at 9 and 14 days of pregnancy. Extracts containing 120 $\mu \mathrm{g}$ of protein were analyzed by immunoblotting with anti-Akt and anti-Erk $1 / 2$ antibodies that either recognize the phosphorylated form (pAkt or pErk1/2) or equally recognize the phosphorylated and dephosphorylated forms, i.e., total levels (Akt and Erk1/2). The blots were also analyzed for phospho-FKHR immunoreactivity. These results are representative of at least two identical experiments. increases throughout all stages of pregnancy and becomes maximal at day 10 of lactation, then decreases during involution (Figure 1d). These results show that PTEN mRNA and protein expression profiles closely correlate throughout development of the mammary gland (Figure 1, compare $\mathrm{c}$ and $\mathrm{d}$ ). To determine whether the transgene was expressed in the expected tissue-specific manner, protein extracts from different tissues of MK-PTEN mice at day 20 of pregnancy and day 10 of lactation were immunoblotted for PTEN. PTEN expression was observed only in the mammary gland (data not shown). This protein expression profile is in agreement with that of other MMTV-driven transgenes (21).

We used immunohistochemistry to localize PTEN within the mammary gland of WT and transgenic mice (Figure 2). In WT mice, PTEN immunostaining is very low in virgin, pregnant, and lactating mice. In 7-week-old virgins, higher PTEN staining is observed in ductal epithelial cells in MK-PTEN as compared with WT littermates (Figure 2). Despite the higher expression of PTEN within the virgin MK-PTEN mammary gland after 6 weeks (Figure 1d), we did not detect any changes in ductal growth or in cellular morphology within the nulliparous mammary gland at the ages of 7 or 9 weeks, as determined by whole mounts and histologically stained paraffin-embedded sections (data not shown). During early pregnancy, late pregnancy, and lactation, about $70 \%, 80 \%$, and $95 \%$ of the lobuloalveoli in MK-PTEN mice were immunoreactive for PTEN, respectively. PTEN staining is very weak in the ductal

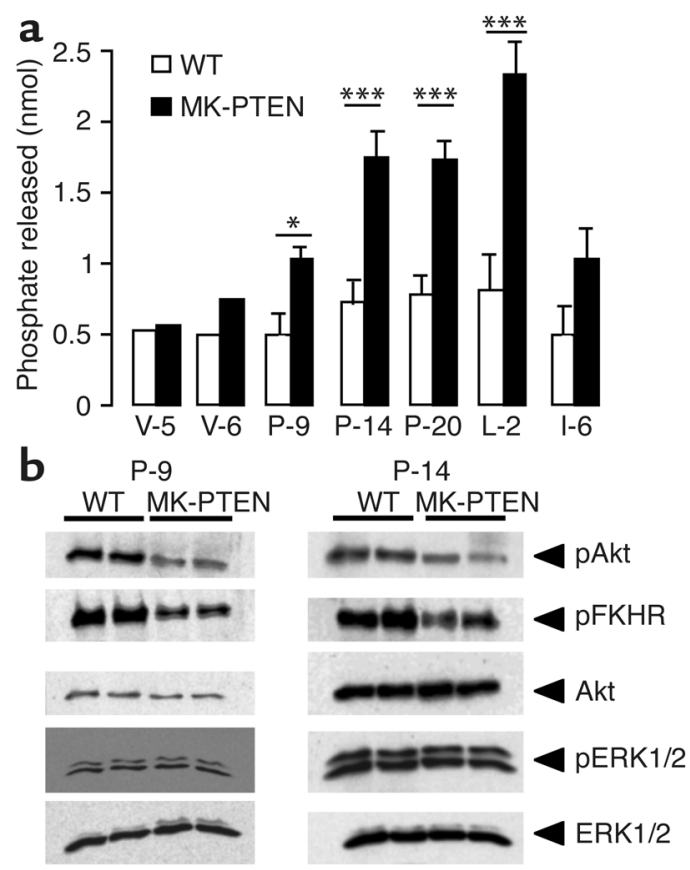


a

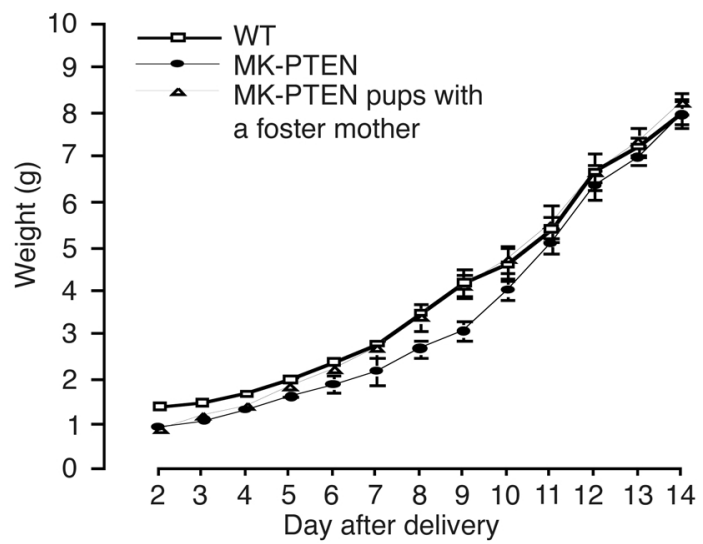

b
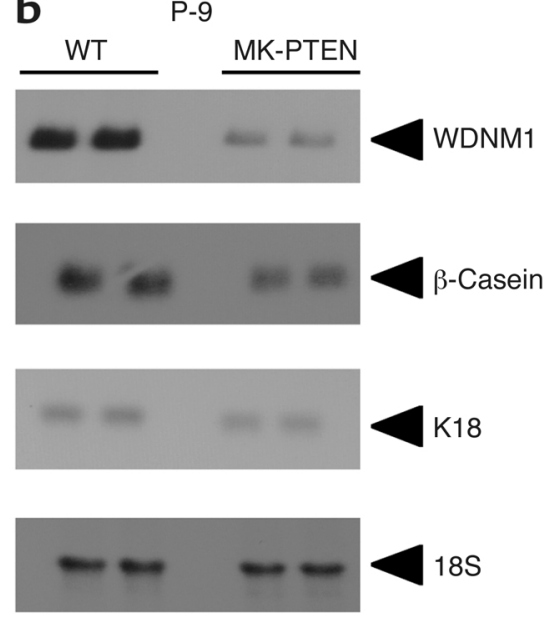

185

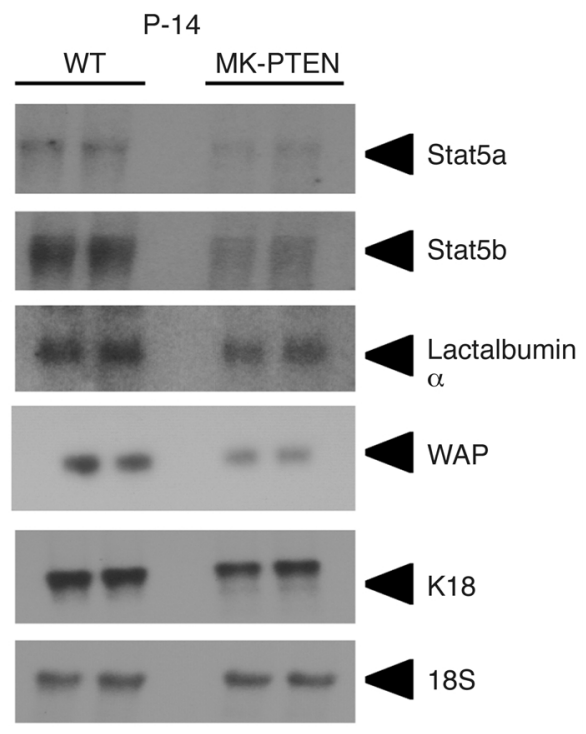

Figure 4

(a) Growth rate of pups from WT and MK-PTEN mice. Newborns from WT and MK-PTEN mothers were weighed each day for 2 weeks. Data are expressed as the mean \pm SD of 16 MK-PTEN pups nursing with either an MK-PTEN or a WT mother, as indicated. (b) Milk protein mRNA expression in mammary glands of MK-PTEN and WT mice. Total RNA was isolated from mammary glands of WT and MK-PTEN at days 9 and 14 of pregnancy. Northern blot analysis was performed using probes corresponding to cDNAs of various milk proteins, as indicated. Blots were stripped and reprobed with an $18 \mathrm{~S}$ probe as a loading control and with $\mathrm{K} 18$ as a marker of epithelial cells. Each lane represents a single animal. Similar results were obtained with a total of four WT and four MK-PTEN mice.

epithelial cells at each of these stages, as shown in Figure 2 , suggesting that overexpression of PTEN in the mammary gland of MK-PTEN mice is more specific to the lobuloalveoli. Thus, the immunohistochemical results are in close agreement with those obtained from PTEN Northern and Western blots.

PTEN phosphatase activity and phosphorylation of Akt and $M A P K$ in the mammary gland of MK-PTEN mice. To determine the functionality of PTEN in the mammary gland of MK-PTEN mice, we measured the PTEN phosphatase activity. We also determined the phosphorylation state of Akt and Erk1/2, which are two known targets of PTEN. PTEN was immunoprecipitated from mammary gland extracts at different stages, and phosphatase activity was measured using PIP3 as substrate. As shown in
Figure 3, PTEN phosphatase activity levels remain relatively constant throughout mammary gland development in WT mice. In striking contrast, PTEN phosphatase activity progressively increases throughout development, pregnancy, and lactation in MK-PTEN mice and decreases after involution (Figure 3a). We also found that during early and middle pregnancy ( 9 and 14 days), the phosphorylation state of Akt and FKHR was decreased by about $50 \%$ in MK-PTEN mice, as compared with WT mice (Figure $3 \mathrm{~b}$ ). However, the level of Erk1/2 phosphorylation was similar in WT and MK-PTEN mice (Figure 3b). Thus, these results suggest that PTEN functions through the PI3K/Akt pathway in the mammary gland of MK-PTEN mice.

Decreases in epithelial cell number and milk protein expression in the mammary gland of MK-PTEN mice are associated with a defect in lactation. The primary biologic function of the mammary gland is to provide nourishment to suckling young in the form of milk. To determine whether PTEN is involved in the functionality of the mammary gland, we examined the ability of MK-PTEN mice to adequately nourish pups (measured by pup weight). We observed that 2 days after the onset of lactation, $30 \%$ of the pups from MK-PTEN females died (data not shown), and the survivors exhibited significant growth retardation until 9 days old (Figure 4a). However, if, immediately after the delivery, WT female mice nursed MK-PTEN pups, the pups survived and grew normally (Figure 4a). These findings suggest that the MK-PTEN newborns are viable but die of malnutrition during nursing. To understand the origin of these physiological perturbations, we performed histological examinations on the mammary glands from MK-PTEN mice. We found that the number of lobuloalveoli in MK-PTEN mice was reduced by $30-50 \%$ in pregnant MK-PTEN mice, as compared with the WT littermates (Figure 2). We also investigated whether these lobuloalveoli were functional by measuring the expression levels of various milk mRNAs during early and middle pregnancy (Figure 4b). At 9 days of pregnancy, WDNM1 and $\beta$-casein mRNA levels were reduced by 
about $40 \%$ in MK-PTEN as compared with WT littermates, as normalized to $18 \mathrm{~S}$ RNA levels. Similar results were observed for the mRNA expression of Stat5a and $5 b$, lactalbumin, and WAP after 14 days of pregnancy (Figure 4b). Keratin 18 serves as a marker of the epithelial cells. No differences in litter size were observed between MK-PTEN and WT mice (data not shown), although MK-PTEN male mice expressed higher levels of PTEN in certain reproductive organs (data not shown).

Mammary epithelium overexpressing PTEN exbibits decreased proliferation and increased apoptosis. The observed decrease in the epithelial content of mammary glands harboring PTEN-overexpressing epithelium may reflect a decrease in the rate of epithelial proliferation and/or an increase in the level of epithelial apoptosis. We tested these two possibilities by quantifying BrdU incorporation and by TUNEL assays, respectively. To assess the levels of epithelial cell proliferation in mice overexpressing PTEN specifically in the mammary gland, mice were labeled with the BrdU. Based on immunohistochemical analysis, the number of BrdU-positive nuclei in mammary epithelium overexpressing PTEN appeared to be lower than that in mammary epithelium of WT mice (Figure 5). To quantify this decrease, the percentage of BrdU-positive nuclei relative to the total number of nuclei was calculated at three different stages of pregnancy (P-9, P-14, and P-20; Figure 5b, left). The percentage of BrdU-positive epithelial nuclei was decreased by approximately $50 \%$ in mammary epithelium overexpressing PTEN compared with that in WT mice. In accordance with these results, we also found that cyclin D1 immunoreactivity was significantly reduced in mammary epithelium of MK-PTEN as compared with WT mice (data not shown). These data demonstrate that the mammary epithelium of MK-PTEN mice is indeed hypoplastic at all stages of pregnancy.

To assess the level of apoptosis in mammary epithelium of MK-PTEN mice, TUNEL analysis was performed on sections prepared from MK-PTEN and WT mice at different stages of pregnancy. TUNEL-positive nuclei were detected by immunohistochemistry. Based on immunohistochemical analysis, the percentage of TUNEL-positive nuclei in mammary epithelium of MK-PTEN mice was approximately two- to threefold greater than that observed in the mammary epithelium of WT mice (Figure 5b, right). Thus, these data suggest that the lower cell density in mammary epithelium of MK-PTEN mice is due to both a decrease in cell proliferation and a concomitant increase in apoptosis.

Profiling gene expression in MK-PTEN transgenic mice. To further understand the developmental defects in the MKPTEN transgenic mice, we profiled the gene expression pattern in mammary tissue 2 days after parturition. Since most genes expressed in mammary tissue are absent from commercial cDNA arrays, we generated an array, the socalled mammochip, that contains 3,600 cDNAs selected from different cDNAs of mammary origin. While 11 genes were expressed preferentially in mammary tissue from MK-PTEN mice, nine genes were preferentially expressed in WT tissue (Table 1). Out of the 11 genes overexpressed in MK-PTEN mammary tissue, six corresponded to known genes. Most notably, the expression of the IGFBP-5 gene was 26 times higher in MK-PTEN mammary tissue. Interestingly, a database search demonstrated that expression of IGFBP-5 is preferentially seen in mammary tumors of transgenic mice. The expression of the carbonic anhydrase 3 was also increased fivefold in MK-PTEN mammary epithelium. The higher expression of both IGFBP-5 and carbonic anhydrase 3 genes in MK-PTEN than in WT mice was confirmed by RT-PCR (Figure 6a). We used immunohistochemistry to localize IGFBP-5 within the mammary gland of WT and MK-PTEN mice (Figure 6b). IGFBP-5 immunostaining is very high in epithelial cells of MK-PTEN mice at day 2 of lactation as compared with the WT littermates (Figure 6b).

Expressed sequence tag database searches demonstrated that the genes overexpressed in MK-PTEN tissue were also highly expressed in mammary tumors of transgenic mice. Three known genes, those encoding

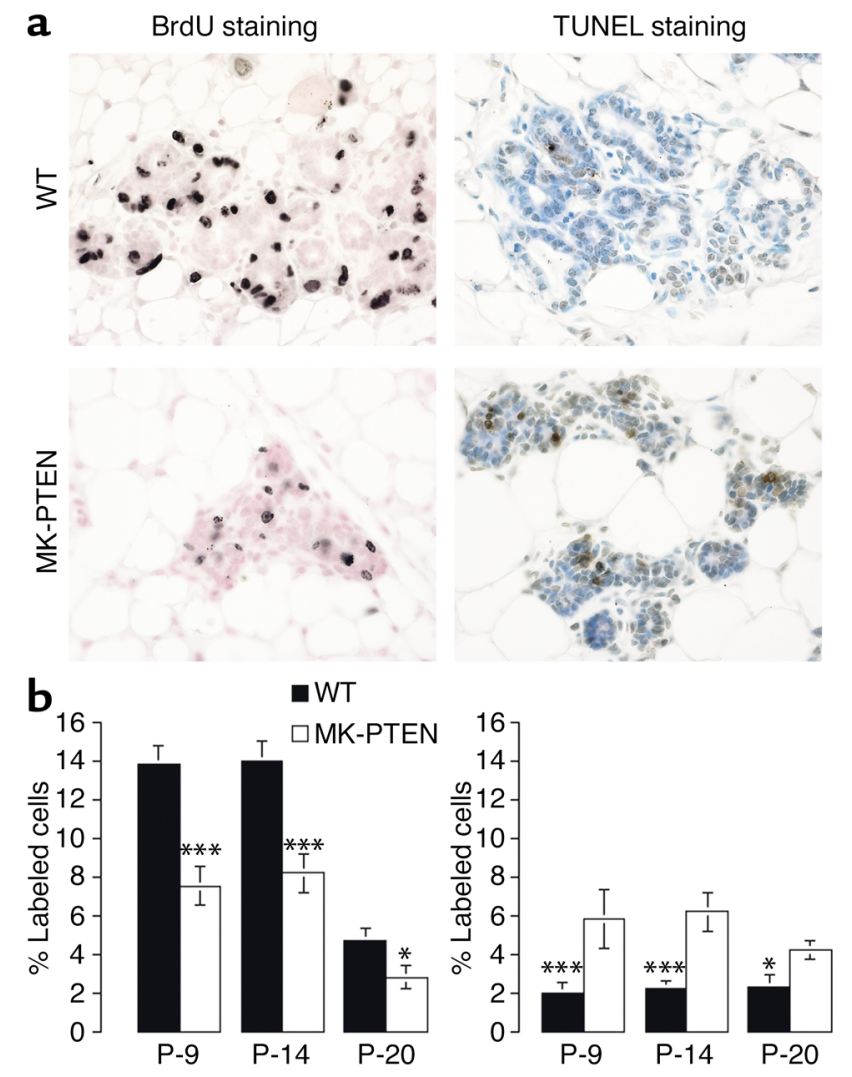

\section{Figure 5}

Cell proliferation is decreased and apoptosis is increased in mammary epithelium from MK-PTEN and WT mice during pregnancy. At the indicated stages, pregnant MK-PTEN and WT mice were injected with BrdU and sacrificed 2 hours later, as described in Methods. (a) BrdU-labeled nuclei were detected by immunostaining, and apoptotic nuclei were stained by TUNEL. (b) About 3,000 nuclei were counted, and the percentage of labeled epithelial cells was determined at 9 (P-9), 14 (P-14), and 20 (P-20) days of pregnancy. Data are expressed as the percentage of labeled cells \pm SD. Averages were compared by $t$ test. Averages were compared by $t$ test. ${ }^{*} P<0.05,{ }^{* * *} P<0.001$. 
Table 1

Genes differentially regulated in mammary tissue of MK-PTEN and WT mice

Genes downregulated in MK-PTEN mice

Image ID

Mammochip

1511382

1511419

1511486

Oncochip

584391

532142

540164

313981

476319

634887

Genes overexpressed in MK-PTEN mice

High-mobility group protein I isoform C DNA-repair protein

GDP dissociation inhibitor 1

Hemoglobin, $\beta$ adult major chain

Glucocorticoid-induced leucine zipper Receptor her3

Mammochip

1196011

948529

1246428

1248573

1314739

864622

Oncochip

469082

582886

351811

517774

391736

472500

\author{
IGFBP-5 \\ Cleavage stimulation factor, $3^{\prime}$ pre-RNA, subunit 3 \\ G0/G1 switch gene 2 \\ General control of amino acid synthesis-like 2 (yeast) \\ Carbonic anhydrase 3 \\ RIKEN cDNA 2310043N10 gene
}

\author{
Kinase PITALRE (cdc2) \\ Complement component 3 \\ Thymosin $\beta 10$ \\ Kinase met \\ Ribosomal protein L44 \\ Cell cycle G0/G1 switch
}

WT/PTEN relative abundance in expressed sequence tag libraries

$\begin{array}{ccccc} & \text { MG virgin } & \text { MG lactating } & \text { MG tumors } & \text { Others } \\ 27 & - & +++ & + & + \\ 15 & - & - & - & ++ \\ 7 & - & ++ & +++ & - \\ & & & & \\ 13 & - & - & - & ++ \\ 9 & - & - & ++ & + \\ 8 & - & - & + & +++ \\ 7 & - & - & - & +++ \\ 4 & - & - & + & ++ \\ 4 & - & - & - & ++ \\ \text { PTEN/WT } & & & & \end{array}$

$\begin{array}{ccccc}26 & + & + & +++ & + \\ 8 & + & + & - & ++ \\ 7 & + & + & +++ & + \\ 5 & - & + & +++ & + \\ 5 & ++ & + & + & - \\ 4 & + & + & + & +++ \\ & & & & \\ 11 & + & + & +++ & ++ \\ 6 & - & - & - & +++ \\ 5 & + & + & + & ++ \\ 4 & + & - & + & +++ \\ 4 & - & + & + & +++ \\ 3 & + & + & +++ & +++\end{array}$

RNA from MK-PTEN and WT mammary tissue at day 2 of lactation was hybridized to the "mammochip" and the "oncochip," and differentially expressed genes were identified as outlined in Methods. The known genes that were differentially expressed are shown and listed by their expression ratio (WT/PTEN and PTEN/WT). In order to evaluate their expression pattern in different tissues, we searched more than 9 million expressed sequence tag clones from GenBank. The relative frequency of hits in cDNA libraries from the mammary glands of virgin (MG virgin) and lactating (MG lactating) mice, in mammary tumors from transgenic mice (MG tumors), and in nonmammary libraries (Others) is shown.

$\delta$-casein, JNK/SAPK-1, and WAP, were expressed 27-, $15-$, and 7-fold more highly, respectively, in WT tissue (Table 1). Again, these results were confirmed by RT-PCR (Figure 6a). The genes and gene products corresponding to the differentially expressed sequence tags have not been investigated. We further monitored the expression of 2,600 known genes, including many oncogenes, on a commercial oncochip. While six genes were expressed preferentially in mammary tissue from MK-PTEN mice, six other genes were preferentially expressed in WT tissue (Table 1). Most of the identified genes were involved in the cell proliferation or apoptosis. Indeed, the $\mathrm{cdc} 2$, complement component 3 , thy$\operatorname{mosin} \beta 10$, and cell cycle G0/G1 genes were expressed 11-, 6-, 5-, and 3-fold more highly, respectively, in MK-PTEN mammary tissue, whereas the high-mobility group protein I isoform C (HMG-IC), glucocorticoid-induced leucine zipper (GILZ), and receptor her3 genes were expressed 13-, 4-, and 4-fold more highly, respectively, in WT mammary tissue (Table 1).

IGFBP-5 is involved in the apoptosis observed in the MK-PTEN mammary epithelial cells. IGFBP-5 is an IGF-binding protein involved in vivo in mammary apoptosis during the involution stage. To determine the role of IGFBP-5 in the apoptosis observed in MK-PTEN mammary epithelium, we isolated and cultured mammary epithelial cells from WT and MK-PTEN mice. After 48 hours of culture, cells were incubated for 24 hours in the presence or absence of an IGFBP-5-blocking peptide, and we determined the apoptosis level by tunnel assay and measured the caspase-3 activity. The percentage of apoptotic cells and the caspase-3 activity were about three and four times higher in MK-PTEN mammary epithelial cells than in WT, respectively (Figure 6c). Interestingly, the IGFBP-5blocking peptide (5 or $10 \mu \mathrm{g} / \mathrm{ml}$ ) reduced by about $40 \%$ $(P<0.05)$ the higher apoptosis level observed in the MKPTEN mammary epithelial cells (Figure 6c). Thus, these results demonstrate that the apoptosis observed in mammary MK-PTEN epithelial cells is due in part to IGFBP-5.

\section{Discussion}

To investigate the role of PTEN in the development of the mammary gland, we created transgenic mice (MK-PTEN) that overexpress an active PTEN specifically in the 
mammary epithelium under the control of the MMTVLTR. MK-PTEN mice overexpress PTEN from puberty, throughout pregnancy and lactation, and during the first 6 days of involution. Ductal development in MK-PTEN mice during puberty was normal, possibly because the level of transgenic PTEN is only twofold higher than in WT littermates. Furthermore, PTEN immunostaining was stronger in the alveolar epithelial cells than in the ductal epithelium of MK-PTEN mammary glands. However, during the early stages of pregnancy, the mammary tissue of MK-PTEN mice exhibits a decrease in the number of alveolar epithelial cells, as compared with that of their WT littermates. This reduction is accompanied by a significant defect in lactation, such that $30 \%$ of newborn pups die of malnutrition during the first 2 days of nursing and the survivors exhibit growth retardation.

Specific proteins have been identified that play important roles during the different stages of mammary gland development (reviewed in ref. 33). Mice deficient in the estrogen receptor exhibit a severe impairment in ductal outgrowth and branching receptor (34), and mice deficient in the progesterone receptor have retarded alveolar development (35). These findings suggest that the estrogen and progesterone receptors play vital roles in mammary gland development during puberty and early pregnancy. Similarly, mammary epithelium does not form in the absence of the prolactin receptor, the transcription factor $\mathrm{C} / \mathrm{EBP} \beta$, the transcription factors Stat $5 \mathrm{a}$ and $5 \mathrm{~b}$, and the LAR phosphatase (reviewed in ref. 24). In $\mathrm{C} / \mathrm{EBP} \beta$-null mice, the deficiency in mammary development is partially a consequence of defective ovarian function (24). Reduced alveolar proliferation during pregnancy was also observed in the MK-PTEN mice. In contrast to the Prolactin receptor- and C/EBP $\beta$-null mice, mammary epithelium in the MK-PTEN mice acquired a partially differentiated state, as indicated by the expression of some milk protein genes. However, loss of normal expression of $\delta$-casein and WAP, as determined by Northern blot and cDNA microarrays, suggests that MK-PTEN mammary did not acquire functional differentiation. In particular, expression of the $\delta$-casein gene reflects the differentiation status of mammary epithelium. While other casein genes are activated around midpregnancy (36), expression of the $\delta$-casein gene occurs at parturition. Thus, the reduced levels of WAP and $\delta$-casein mRNA in MK-PTEN tissue reflect that this tissue has not undergone full differentiation. Furthermore, the expression of the carbonic anhydrase found preferentially in mammary stroma was significantly higher in MK-PTEN mammary tissue, reflecting again a reduced alveolar development. Thus, the tumor suppressor PTEN protein appears to play a critical role in the control of mammary cell proliferation, and to some extent in cell differentiation.

Several studies show that the proliferation of glioblastoma cell lines and breast cancer cell lines is significantly inhibited by the overexpression of PTEN (reviewed in ref. 37). This antiproliferative effect is dependent on the lipid phosphatase activity of PTEN. In the mammary tissue of MK-PTEN mice, we show that the reduction of epithelial cell proliferation is paralleled by a decrease in the phosphorylation state of Akt. To date, PTEN is the only known enzyme that dephosphorylates the $\mathrm{D} 3$ position in inositol phospholipids, suggesting that PTEN may function to oppose PI3K and Akt signaling pathways. It has been shown that constitutively active forms of PI3K and Akt can rescue PTEN-induced suppression of cell proliferation, suggesting that an Akt-mediated signaling plays a major role in PTEN-sensitive cell proliferation (38). The MAPK cascade is another growth factor-mediated signaling pathway. MAPK-mediated growth signaling can oppose the effects of PTEN overexpression on proliferation or migration in different cell lines (39). However, it has also been shown that overexpression of PTEN markedly reduces the phosphorylation of Erk2 in U87MG cells (13). This inhibition of MAPK by PTEN could be attributable to effects of She
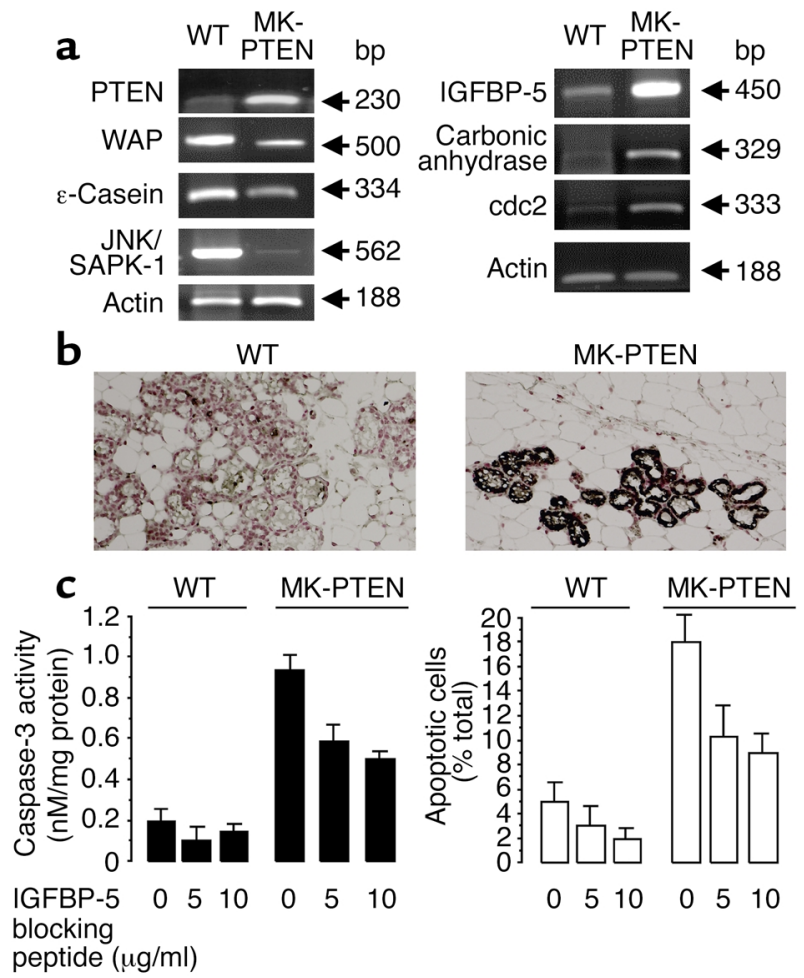

\section{Figure 6}

(a) mRNA expression determined by RT-PCR of some genes up- or downregulated in mammary tissue of MK-PTEN mice as compared with that of WT mice. These results are representative of two WT and two MK-PTEN mice different from those used to perform the microarray experiment. (b) Immunohistochemical localization of IGFBP-5 in the mammary gland of MK-PTEN and WT mice. Sections from mammary glands removed from mice lactating for 2 days were analyzed by immunohistochemistry as described in Methods. (c) An IGFBP-5blocking peptide reduces the level of the apoptotic mammary epithelial cells of MK-PTEN mice. Following incubation of mammary epithelial cells from WT and MK-PTEN mice in DMEM with serum for 48 hours, the cells were untreated or treated with 5 or $10 \mu \mathrm{g} / \mathrm{ml}$ of an IGFBP-5-blocking peptide. Apoptotic cells were detected by TUNEL assay and caspase- 3 activity was determined by the ApoAlert colorimetric assay as described in Methods. The data are representative of three independent experiments performed in triplicates. 
(13) or effects on IRS-1 (40). However, in vivo analyses of cells from PTEN knockout embryos fail to show changes in basal Erk activity (41). In MK-PTEN and WT mice, the level of MAPK (Erk1/2) phosphorylation in the mammary epithelium is similar during pregnancy despite the observed reduction in the lobuloalveolar epithelial cells. Using the mammary-enriched cDNA microarray, we showed that the expression of the JNK/SAPK-1 gene was 15 times lower in MK-PTEN mammary tissue. Furthermore, we have confirmed this result by Western blot at the protein level (data not shown). Some reports showed that the activation of the JNK kinase in response to prolactin increases cell proliferation of mammary epithelial cells (42). Thus, the lower cell proliferation of mammary epithelial cells of MK-PTEN could be explained by a marked reduction of the activation of Akt but also by a defect of other signaling pathways such as the JNK/SAPK-1.

Using an oncogene-enriched cDNA microarray, we also identified some genes, including HMG-IC, cdc2, and the cell cycle G0/G1 switch, that are differentially expressed in the MK-PTEN and WT mammary tissue. HMG-IC is an abundant component of rapidly proliferating undifferentiated cells, and high-level expression of HMG-IC is also observed in diverse tumors (reviewed in ref. 43). Thus, the differential expression of these genes could be also related to the reduction of the epithelial cell proliferation observed in the MK-PTEN mammary tissue. Cyclin-dependent kinase (CDK) inhibitors such as $\mathrm{p} 21, \mathrm{p} 57$, and $\mathrm{p} 27$ are thought to play a role in PTEN-mediated inhibition of cell proliferation (44). We did not detect changes in p27, p21, and p57 expression levels in the mammary glands of MK-PTEN mice on day 2 of lactation, when PTEN overexpression is very high (data not shown). One possible explanation for this negative finding is that in some cell types, PTEN overexpression does not affect the protein levels of p27 but rather changes the activity of the CDK2/p27 complex. Other studies have demonstrated an involvement of $\mathrm{pRb}$ phosphorylation in PTEN-induced cell cycle arrest (38). It has also been shown that overexpression of PTEN downregulates cyclin D1 (45). We did observe a reduction in cyclin D1 levels in the mammary tissue of MK-PTEN mice (data not shown).

In mammary epithelium of MK-PTEN mice, the levels of apoptosis were two- to threefold higher, which agrees with findings that PTEN-null mouse embryo fibroblasts are highly resistant to apoptotic stimuli (41). Using the Cre-loxP system to generate a $\mathrm{T}$ cell-specific deletion of the PTEN gene, Suzuki et al. show that the $\mathrm{T}$ cells $\left(\mathrm{PTEN}^{-/-}\right)$hyperproliferate and resist apoptosis (46). Fas-induced apoptosis of peripheral $\mathrm{T}$ and $\mathrm{B}$ cells is also decreased in $\mathrm{PTEN}^{+/-}$mice (47). In mammary epithelium of MK-PTEN mice, the phosphorylation state of Akt is decreased and the level of lipid phosphatase activity associated with PTEN is increased. This may explain the higher level of apoptosis observed in these animals as compared with their WT littermates. From these results, it would be predicted that changes in PTEN activity, through overexpression or deletion, could alter the sensitivity of cells to apoptosis.

Using mammary-enriched cDNA microarrays to examine changes in gene expression profile in MK-PTEN as compared with WT mice, we show that MK-PTEN mice expressed IGFBP-5 26 times more highly than did the WT littermates 2 days after parturition. Furthermore, using primary culture of mammary epithelial cells, we showed that an IGFBP-5-blocking peptide reduces significantly the level of apoptosis as well as the caspase-3 activity observed in the mammary epithelial cells from the MK-PTEN mice. These results suggest strongly that the higher level of apoptosis in the mammary gland of MK-PTEN is partially due to the higher expression of IGFBP-5. IGFBP-5 is one of the major factors involved in mammary apoptosis in vivo (48). In vitro, in mammary epithelial cells in primary culture, lumen formation and DNA laddering indicative of apoptosis are also associated with an increase in the IGFBP-5 levels (49).

Using an oncogene-enriched cDNA microarray, we identified other genes involved in the cell survival or apoptosis. Amongst these genes, some were highly expressed in MK-PTEN mammary tissue. These genes include the thymosin $\beta 10$ (50) and the complement component 3 (51). GILZ is reported to inhibit the expression of Fas ligand (52), which is a mediator of apoptosis of mammary epithelial cells during the first stage of mammary involution (53). Our data show a lower expression of the GILZ gene in MK-PTEN mammary tissue, suggesting a lower inhibition of Fas and consequently a higher sensibility to the apoptosis. Thus, our results suggest that the higher apoptosis observed in the MK-PTEN mammary tissue could be due to a decrease of Akt activation but also an induction or repression of some genes directly involved in the apoptosis process.

In conclusion, overexpression of active PTEN in mammary alveolar epithelium causes a marked reduction in cell proliferation, a marked increase in apoptosis, and an incomplete functional differentiation, which results in lactation failure. This suggests that the tumor suppressor protein PTEN can control mammary gland development; furthermore, an increase in proteins such as IGFBP-5 or a reduction in JNK/SAPK-1 may be directly responsible for this effect. The specific inactivation of the PTEN gene in mouse mammary epithelium will be needed to confirm our transgenic dominant gain-of-function experiments. Future studies with MK-PTEN mice will allow us to establish whether the high level of PTEN expression in the mammary epithelium is sufficient to reduce mammary tumor formation and/or progression.

\section{Acknowledgments}

We thank Keiko Miyoshi for advising us on mammary gland biopsy techniques and Jonathan Shillingford for guidance with immunohistochemistry. We thank Charis Eng for providing the PTEN mAb 6H2.1. We also thank Gertraud W. Robinson and Shoshana Yakar for advice and helpful discussions, and Dana Beitner-Johnson for 
editing the manuscript and for critical comments. This work was supported in part by a grant from Institut National de la Recherche Agronomique (INRA; France) (to Joëlle Dupont while she was on leave from INRA).

1. Hennighausen, L., and Robinson, G.W. 1998. Think globally, act locally: the making of a mouse mammary gland. Genes Dev. 12:449-455.

2. Forsyth, I.A. 1989. Growth factors in mammary gland function. J. Reprod. Fertil. 85:759-770.

3. Lyons, W.R., Li, C.H., and Johnson, R.F. 1958. The hormonal control of mammary growth and lactation. Recent Prog. Horm. Res. 14:219-254.

4. Luetteke, N.C., et al. 1999. Targeted inactivation of the EGF and amphiregulin genes reveals distinct roles for EGF receptor ligands in mouse mammary gland development. Development. 126:2739-2750.

5. Xie, W., Paterson, A.J., Chin, E., Nabell, L.M., and Kudlow, J.E. 1997. Targeted expression of a dominant negative epidermal growth factor receptor in the mammary gland of transgenic mice inhibits pubertal mammary duct development. Mol. Endocrinol. 11:1766-1781.

6. Hadsell, D.L., and Bonnette, S.G. 2000. IGF and insulin action in the mammary gland: lessons from transgenic and knockout models. J. Mam mary Gland Biol. Neoplasia. 5:19-30.

7. Weber, M.S., et al. 1998. Expression of ovine insulin-like growth factor1 (IGF-1) stimulates alveolar bud development in mammary glands of transgenic mice. Endocrine. 8:251-259.

8. Hadsell, D.L., Greenberg, N.M., Fligger, J.M., Baumrucker, C.R., and Rosen, J.M. 1996. Targeted expression of des(1-3) human insulin-like growth factor $I$ in transgenic mice influences mammary gland development and IGF-binding protein expression. Endocrinology. 137:321-330.

9. Neuenschwander, S., et al. 1996. Involution of the lactating mammary gland is inhibited by the IGF system in a transgenic mouse model. J. Clin. Invest. 97:2225-2232.

10. Steck, P.A., et al. 1997. Identification of a candidate tumour suppressor gene, MMAC1, at chromosome $10 \mathrm{q} 23.3$ that is mutated in multiple advanced cancers. Nat. Genet. 15:356-362.

11. Li, J., et al. 1997. PTEN, a putative protein tyrosine phosphatase gene mutated in human brain, breast, and prostate cancer. Science. 275:1943-1947.

12. Yamada, K.M., and Araki, M. 2001. Tumor suppressor PTEN: modulator of cell signaling, growth, migration and apoptosis. J. Cell Sci. 114:2375-2382.

13. Gu, J., Tamura, M., and Yamada, K.M. 1998. Tumor suppressor PTEN inhibits integrin- and growth factor-mediated mitogen-activated protein (MAP) kinase signaling pathways. J. Cell Biol. 143:1375-1383.

14. Tamura, M., Gu, J., Takino, T., and Yamada, K.M. 1999. Tumor suppressor PTEN inhibition of cell invasion, migration, and growth: differential involvement of focal adhesion kinase and p130Cas. Cancer Res. 59:442-449.

15. Simoncini, T., et al. 2000. Interaction of oestrogen receptor with the regulatory subunit of phosphatidylinositol-3-OH kinase. Nature. 407:538-541.

16. Goupille, O., Barnier, J.V., Guibert, B., Paly, J., and Djiane, J. 2000. Effect of PRL on MAPK activation: negative regulatory role of the C-terminal part of the PRL receptor. Mol. Cell. Endocrinol. 159:133-146.

17. Liang, L., Jiang, J., and Frank, S.J. 2000. Insulin receptor substrate-1mediated enhancement of growth hormone-induced mitogen-activated protein kinase activation. Endocrinology. 141:3328-3336.

18. Faraldo, M.M., Deugnier, M.A., Thiery, J.P., and Glukhova, M.A. 2001. Growth defects induced by perturbation of $\beta 1$-integrin function in the mammary gland epithelium result from a lack of MAPK activation via the Shc and Akt pathways. EMBO Rep. 2:431-437.

19. Finlay, D., et al. 2000. MAP kinase pathway signalling is essential for extracellular matrix determined mammary epithelial cell survival. Cell Death Differ. 7:302-313.

20. Strange, R., Metcalfe, T., Thackray, L., and Dang, M. 2001. Apoptosis in normal and neoplastic mammary gland development. Microsc. Res. Tech. 52:171-181.

21. Hutchinson, J., Jin, J., Cardiff, R.D., Woodgett, J.R., and Muller, W.J. 2001. Activation of Akt (protein kinase B) in mammary epithelium provides a critical cell survival signal required for tumor progression. Mol. Cell. Biol. 21:2203-2212

22. Schwertfeger, K.L., Richert, M.M., and Anderson, S.M. 2001. Mammary gland involution is delayed by activated akt in transgenic mice. Mol. Endocrinol. 15:867-881.

23. Ma, Z.Q., Chua, S.S., DeMayo, F.J., and Tsai, S.Y. 1999. Induction of mammary gland hyperplasia in transgenic mice over-expressing human Cdc25B. Oncogene. 18:4564-4576.

24. Enomoto, K., Furuya, K., Maeno, T., Edwards, C., and Oka, T. 1987. Mechanically induced electrical responses in murine mammary epithelials cells in primary culture. FEBS Lett. 223:82-86.
25. Rees, C., and Clemmons, D.R. 1998. Inhibition of IGFBP-5 binding to extracellular matrix and IGF-1 stimulated DNA synthesis by a peptide fragment of IGFBP-5. J. Cell. Biochem. 3:375-381.

26. Robinson, G.W., Johnson, P.F., Hennighausen, L., and Sterneck, E. 1998. The C/EBPbeta transcription factor regulates epithelial cell proliferation and differentiation in the mammary gland. Genes Dev. 12:1907-1916.

27. Dupont, J., Derouet, M., Simon, J., and Taouis, M. 1998. Nutritional state regulates insulin receptor and IRS-1 phosphorylation and expression in chicken. Am. J. Physiol. 274:E309-E316.

28. Georgescu, M., Kirsch, K.H., Akagi, T., Shishido, T., and Hanafusa, H. 1999. The tumor-suppressor activity of PTEN is regulated by its carboxyl-terminal region. Proc. Natl. Acad. Sci. USA. 96:10182-10187.

29. Perren, A., et al. 1999. Immunohistochemical evidence of loss of PTEN expression in primary ductal adenocarcinomas of the breast. Am. J. Pathol. 155:1253-1260.

30. Desai, K.V., et al. 2002. Initiating oncogenic event determines geneexpression patterns of human breast cancer models. Proc. Natl. Acad. Sci. USA. 99:6967-6972.

31. Eisen, M.B., and Brown, P.O. 1999. DNA arrays for analysis of gene expression. Methods Enzymol. 303:179-205.

32. mAdb System Home Page. http://nciarray.nci.nih.gov.

33. Hennighausen, L., and Robinson, G.W. 2001. Signaling pathways in mammary gland development. Dev. Cell. 1:467-475.

34. Bocchinfuso, W.P., and Korach, K.S. 1997. Mammary gland development and tumorigenesis in estrogen receptor knockout mice. J. Mammary Gland Biol. Neoplasia. 2:323-334.

35. Lydon, J.P., et al. 1995. Mice lacking progesterone receptor exhibit pleiotropic reproductive abnormalities. Genes Dev. 9:2266-2278.

36. Burdon, T., Sankaran, L., Wall, R.J., Spencer, M., and Hennighausen, L. 1991. Expression of a whey acidic protein transgene during mammary development. Evidence for different mechanisms of regulation during pregnancy and lactation. J. Biol. Chem. 266:6909-6914.

37. Maehama, T., Taylor, G.S., and Dixon, J.E. 2001. PTEN and myotubularin: novel phosphoinositide. Annu. Rev. Biochem. 70:247-279.

38. Paramio, J.M., Navarro, M., Segrelles, C., Gomez-Casero, E., and Jorcano, J.L. 1999. PTEN tumour suppressor is linked to the cell cycle control through the retinoblastoma protein. Oncogene. 18:7462-7468.

39. Davies, M.A., et al. 1999. Regulation of Akt/PKB activity, cellular growth, and apoptosis in prostate carcinoma cells by MMAC/PTEN. Cancer Res. 59:2551-2556.

40. Weng, L.P., Smith, W.M., Brown, J.L., and Eng, C. 2001. PTEN inhibits insulin-stimulated MEK/MAPK activation and cell growth by blocking IRS-1 phosphorylation and IRS-1/Grb2/Sos complex formation in a breast cancer model. Hum. Mol. Genet. 10:605-616.

41. Stambolic, V., et al. 1998. Negative regulation of PKB/Akt-dependent cell survival by the tumor suppressor PTEN. Cell. 95:29-39.

42. Olazabal, I., Munoz, J., Ogueta, S., Obregon, E., and Garcia-Ruiz, J.P. 2000. Prolactin-PRL receptor system increases cell proliferation involving JNK (c-jun amino terminal kinase) and AP-1 activation: inhibition by glucocorticoids. Mol. Endocrinol. 14:564-575.

43. Goodwin, G. 1998. The high mobility group protein, HMGI-C. Int. J. Biochem. Cell Biol. 30:761-766.

44. Wu, R.C., Li, X., and Schonthal, A.H. 2000. Transcriptional activation of p21WAF1 by PTEN/MMAC1 tumor suppressor. Mol. Cell. Biochem. 203:59-71.

45. Weng, L.P., Brown, J.L., and Eng, C. 2001. PTEN coordinates G(1) arrest by down-regulating cyclin D1 via its protein phosphatase activity and up-regulating p27 via its lipid phosphatase activity in a breast cancer model. Hum. Mol. Genet. 10:599-604.

46. Suzuki, A., et al. 2001. T cell-specific loss of Pten leads to defects in central and peripheral tolerance. Immunity. 14:523-534.

47. Di Cristofano, A., et al. 1999. Impaired Fas response and autoimmunity in Pten+/- mice. Science. 285:2122-2125.

48. Tonner, E., et al. 2000. Insulin-like growth factor binding protein-5 (IGFBP-5) potentially regulates programmed cell death and plasminogen activation in the mammary gland. Adv. Exp. Med. Biol. 480:45-53.

49. Chen, J.C., et al. 1994. Insulin-like growth factor-binding protein enhancement of insulin-like growth factor-I (IGF-I) mediated DNA synthesis and IGF-I binding in a human breast carcinoma cell line. J. Cell. Physiol. 158:69-78.

50. Gutierrez-Pabello, J.A., McMurray, D.N., and Adams, L.G. 2002. Upregulation of thymosin beta 10 by Mycobacterium bovis infection of bovine macrophages is associated with apoptosis. Infect. Immun. 70:2121-2127.

51. Connolly, A.M., Keeling, R.M., Streif, E.M., Pestronk, A., and Mehta, S. 2002. Complement 3 deficiency and oral prednisolone improve strength and prolong survival of laminin alpha2-deficient mice. J. Neuroimmunol. 127:80-87.

52. Mittelstadt, P.R., and Ashwell, J.D. 2001. Inhibition of AP-1 by the glucocorticoid-inducible protein GILZ. J. Biol. Chem. 276:29603-29610.

53. Song, J., et al. 2000. Roles of Fas and Fas ligand during mammary gland remodeling. J. Clin. Invest. 106:1209-1220. 